\title{
Importancia del uso de las TIC en los procesos de formación integral de la infancia*
}

\author{
Edith Recalde España** \\ Blanca Nery Serna Agudelo*** \\ Sandra Liliana Stella Polo****
}

Fecha de recepción: 26 de abril de 2015

Fecha de revisión: 17 de mayo de 2015

Fecha de aprobación: 2 de junio de 2015

\section{Resumen}

La urgencia que tienen las instituciones educativas del país con respecto a incorporar acciones pedagógicas y didácticas concretas a sus currículos, y que den respuesta a la necesidad de ubicar los procesos de enseñanza y aprendizaje de la infancia de

* Artículo de investigación científica y tecnológica. Investigación-acción pedagógica. Fecha de inicio: noviembre de 2011. Fecha de culminación: junio de 2014. Resultados de un proyecto de investigación adscrito a la Vicerrectoría de Investigaciones - Universidad de la Amazonia.

** Licenciada en Lingüística y Literatura, especialista en Pedagogía de la Universidad de la Amazonia, magíster en Educación, Fundación Universitaria de Oriente, en convenio con la fundación Universitaria del Norte, Medellín. Grupo de investigación: Pedagogía e Infancia, Línea de Investigación LINFA. Universidad de la Amazonia. Dirección de correspondencia: Calle 17 diagonal 17 carrera 3F, barrio Porvenir. Departamento de Educación a Distancia, docente. Correo electrónico: e.recalde@udla. edu.co

*** Licenciada en Lingüística y Literatura, especialista en Educación Básica con énfasis en Procesos Pedagógicos de la Universidad de la Amazonia, magíster en Educación con énfasis en Resolución de Conflictos en el Ámbito Escolar, Universidad de Aconcagua, Chile. Grupo de investigación: Pedagogía e Infancia, Línea de Investigación LINFA. Universidad de la Amazonia. Dirección de correspondencia: Calle 17 diagonal 17 carrera 3F, barrio Porvenir. Programa Licenciatura en Pedagogía Infantil, docente. Correo electrónico: b.serna@udla.edu.co, blancanerys@hotamil.com

$* * * *$ Ingeniera de Sistemas, Universidad Distrital Francisco José de Caldas; especialista en Pedagogía de la Universidad de la Amazonia. Grupo de investigación: Pedagogía e Infancia, Línea de Investigación LINFA. Universidad de la Amazonia. Dirección de correspondencia: Calle 17 diagonal 17 carrera 3F, barrio Porvenir. Programa Licenciatura en Pedagogía Infantil. Coordinadora del programa. Correo electrónico: s.stella@udla.edu.co 
acuerdo a los avances y las exigencias de la era de la comunicación y la información o era digital, se constituye en una de las principales razones que ha motivado la presente investigación. Otra, parte de la hipótesis corresponde a que en la actualidad en el departamento del Caquetá no se está logrando dar una respuesta efectiva a esta necesidad latente, aun cuando existen en algunas instituciones las condiciones mínimas de infraestructura para ello.

El objetivo de esta investigación se enfoca en identificar la manera en que las TIC están siendo articuladas a los procesos de formación de la infancia en cinco instituciones educativas del departamento del Caquetá. Teniendo en cuenta algunas condiciones para su desarrollo, el contexto y el marco académico en el que está inserta, se optó por desarrollarla desde la investigación-acción pedagógica con el énfasis en la práctica pedagógica del maestro Stenhouse (1981). El marco teórico se fundamenta en estudios e investigaciones relacionadas con la incorporación de las TIC al aula escolar que han venido desarrollando pedagogos, psicólogos y científicos destacados tales como: Crook (1998); Papert (1995), (1997); Tapscott (1998); Prensky (2001); Buckingham (2002); Barbero (2000), Escudero (2001), Tedesco (2000), Valenzuela (2004), entre otros.

\section{Palabras clave}

TIC, infancia, desarrollo integral, proyecto de aula, contexto.

\section{The importance of the use of ict for integral education processes in childhood}

\section{Abstract}

The urgency that have educational institutions in the country with respect to incorporate specific pedagogical and educational actions to their resumes, and that respond to the need to locate the processes of teaching and learning of children according to the progress and the requirements of the era of information and communication or it was digital, it constitutes one of the main reasons which led to this research. Moreover, one of the hypothesis that currently in the Department 
of Caquetá is failing to give an effective response to this latent need, even though there are some minimum conditions of infrastructure institutions for this purpose.

The objective of this research is focused on identifying the way in which ICT are being articulated to the processes of formation of children in five educational institutions in the Department of Caquetá. Taking into account, some conditions for its development, the context and the academic framework in which is inserted, is opted to develop it from pedagogical action research with emphasis on the pedagogical practice of the master, Stenhouse (1981). The theoretical framework is based on studies and research related to the incorporation of ICT to the classroom that teachers have been developing psychologists and scientists such as: Crook (1998); Papert (1995), (1997); Tapscott (1998); Prensky (2001); Buckingham (2002); Barber (2000), Squire (2001), Tedesco (2000), Valenzuela (2004), among others.

\section{Keywords}

TIC, childhood, integral development, Classroom project, context.

\section{Importância do uso das tic nos processos de formação integral da infância}

\section{Resumo}

A urgência que têm instituições educacionais do país no que diz respeito a acções específicas de pedagógicas e educativas incorporadas a seus currículos, e que responde à necessidade de localizar os processos de ensino e aprendizagem das crianças de acordo com o progresso e as exigências da era da informação e comunicação ou era digital, constitui um dos principais motivos que levaram a esta pesquisa. Outra parte da hipótese é que hoje no departamento de Caquetá é está falhando ao dar uma resposta eficaz a esta necessidade latente, embora existam algumas condições mínimas das instituições de infra-estrutura para esta finalidade.

O objetivo desta pesquisa é focado em identificar a maneira em que as TIC estão sendo articulados aos processos de formação das crianças em cinco instituições de ensino no departamento de Caquetá. Tendo em conta, algumas condições para o seu desenvolvimento, o contexto e a estrutura acadêmica em que está inserido, é 
optou por desenvolvê-lo de pesquisa-ação pedagógica com ênfase sobre a prática pedagógica do mestre, Stenhouse (1981). O referencial teórico baseia-se em estudos e pesquisas relacionadas com a incorporação das TIC para a sala de aula que os professores foram desenvolvendo psicólogos e cientistas tais como: Crook (1998); Papert (1995), (1997); Tapscott (1998); Prensky (2001); Buckingham (2002); Barbeiro (2000), Squire (2001), Tedesco (2000), Vaz (2004), entre outros.

\section{Palavras-Chave}

TIC, infância, contexto de desenvolvimento integral, projeto de sala de aula..

\section{Introducción}

El tema central abordado en el proyecto de investigación con respecto a la importancia del uso de las TIC en los procesos de formación integral de la infancia, tiene un nivel de importancia de orden nacional y local, en el sentido que socializa los hallazgos obtenidos en la fase diagnóstica; en la que se logró conocer las dinámicas e intencionalidades institucionales a través de las cuales los docentes del preescolar en el grado Transición, hacen uso de las tecnologías de la información y la comunicación en la cotidianidad del aula; de igual forma, muestra el proceso de acompañamiento pedagógico (fase de implementación) a las instituciones educativas del contexto local para el conocimiento respecto a la importancia que le dan a la utilización de las TIC como estrategia pedagógica, de la cual se derivan posteriormente jornadas de asesoría con relación a la fundamentación y diseño del proyecto pedagógico de aula, como estrategia didáctica que favorece la inclusión de las TIC en los procesos de formación integral de la infancia, en correspondencia con los planes de estudio de cada una de las instituciones educativas focalizadas en el sector urbano y rural del municipio de Florencia y el departamento del Caquetá (Colombia).

De igual forma, este artículo enfatiza en la importancia que tiene para el mejoramiento de los procesos educativos establecer escenarios de socialización e interacción con otras comunidades educativas, y por esta razón muestra la importancia del establecimiento de redes de apoyo para socializar ante otras comunidades tanto nacionales como internacionales, los procesos que desarrollan actualmente las instituciones educativas y que requieren mejoramiento, y por ello se dan a conocer las 
dinámicas a través de las cuales se pretende mejorar la incorporación de las TIC a los procesos de formación de los niños en preescolar, grado Transición, en procura de generar aprendizajes mayormente significativos.

Esta actividad se desarrolló a través de la ponencia realizada en el marco de un simposio a nivel internacional, donde se hizo especial relevancia acerca de la importancia de la integración de las TIC en los procesos de formación de la infancia como una de las principales necesidades en el contexto de la educación infantil, teniendo en cuenta los estándares de competencias, los lineamientos curriculares del nivel preescolar reglamentados desde el Ministerio de Educación Nacional, para garantizar la formación integral de la primera infancia en correspondencia con los retos y desafíos de la era de la información y la comunicación.

Asimismo, se contribuyó al mejoramiento de las prácticas pedagógicas con respecto a los procesos de enseñanza y aprendizaje en el nivel preescolar, propiciando el desarrollo de capacidades y habilidades para hacer uso pedagógico, adecuado y responsable de las TIC. De igual manera, sensibilizar a los docentes y padres de familia respecto a la importancia del tema de este proyecto como factor esencial en la formación integral de niños y niñas.

En correspondencia con el documento Ruta Maestra, se expresa la necesidad de orientar el uso didáctico de las nuevas tecnologías y la integración de la tecnología en el currículo; para lograr tal propósito, invita a todos los docentes en ejercicio y en procesos de formación a emprender el camino hacia el cambio en la práctica docente de tal manera que permita:

Transformar las instituciones educativas de manera progresiva y con un plan que no incluya solamente la dotación de equipos y de conectividad, sino que promueva la capacitación permanente del docente en el uso y manejo de las soluciones tecnológicas como estrategia didáctica de aprendizaje (Hernández, 2012, p. 3).

En tal sentido, la Licenciatura en Pedagogía Infantil modalidad a Distancia de la Universidad de la Amazonia, identificó la necesidad de investigar acerca del uso de las TIC en los procesos de formación integral de la infancia, teniendo como fuente de información los trabajos integrados finales TIF "Proyecto de investigación que da respuesta al eje problémico que integra todas las unidades temáticas de un bloque programático"; para este caso, el tercer semestre, a partir de los cuales se realizó un diagnóstico de la sistematización de dichos proyectos haciendo uso de 
la rejilla "síntesis de resultados pregunta de investigación" que alimenta la línea de investigación en infancia LINFA.

El proceso de sistematización hace parte del proyecto de investigación denominado Sistematización de Experiencias Pedagógicas y Prácticas Investigativas en y desde la Licenciatura en Pedagogía Infantil, liderado por el grupo de investigación Pedagogía e Infancia, registrado en la plataforma ScienTi de Colciencias. Documento maestro Licenciatura en Pedagogía Infantil (Celi Martínez Elvia Helena et al., 2012).

Por consiguiente, en el campo de la inclusión de las TIC en el aula infantil en el Caquetá, se optó por considerar y tomar como punto de referencia la información relevante contenida en dichos trabajos; además, desde la unidad temática denominada Educación y Comunicación, se aborda específicamente el uso de los medios y las nuevas tecnologías en la escuela con intencionalidad pedagógica, como lo afirma Barbero (2005). En este sentido, particularmente en el aula infantil como estrategia pedagógica desde unas orientaciones teóricas y metodológicas, con perspectiva crítica y transformadora, como lo manifiesta Prensky (2001).

En relación con el análisis realizado se determinaron los criterios de investigación egresados y contexto que permitieron concretar las instituciones educativas focalizadas en el municipio de Florencia y el departamento de Caquetá. Respecto a la categoría contexto; se unifica el criterio para la población estudiantil rural y urbana, las cuales se encuentran en condiciones de vulnerabilidad, unas más que otras, en el sentido que las comunidades mayormente afectadas están ubicadas estratégicamente agrupadas en poblaciones provenientes de barrios habitados por familias de escasos recursos económicos, las cuales generan su sustento a partir de trabajos informales y oficios varios.

En general, las condiciones de vida de los niños se reflejan en la escuela en: bajo rendimiento académico, desinterés y desmotivación en la realización de actividades académicas de refuerzo en casa Tedesco (2000); por consiguiente, esta situación en la mayoría de las veces incide en escaso contacto de los niños con medios tecnológicos apropiados para promover y acompañar el desarrollo del aprendizaje Tapscott (1998). Situación que conlleva al desconocimiento de los retos y desafíos que propone la era de la información y la comunicación en el sentido que: 
Mediante el uso de las TIC se garantiza más calidad (capacidad de atender a la diversidad), más eficiencia (optimización en el uso de recursos) y mayor equidad (asegurar iguales oportunidades para todos). Al cumplir con estos requisitos y garantías la educación se convierte, de manera efectiva, en el mecanismo más relevante y eficaz a favor de la inclusión social, el desarrollo y la mejora de la competitividad (Carneiro, 2010).

Por otro lado, la categoría de investigación relacionada con egresados, contempla la participación activa en calidad de auxiliares de investigación o como coinvestigadores, como una oportunidad de vinculación a la docencia; constituyéndose como un factor favorable en el desarrollo de esta investigación. De esta manera, con los anteriores criterios de investigación, se vinculan instituciones educativas ubicadas en el contexto urbano y rural, la cual se constituye en uno de los objetivos de la investigación para la fase diagnóstica. En consecuencia, las instituciones educativas focalizadas son:

- Municipio de Florencia: Institución Educativa Instituto Técnico Agroindustrial de la Amazonia, sede Obrero (urbano), Institución Educativa Nueva Jerusalén (rural).

- Departamento del Caquetá: Municipio de La Montañita, Institución Educativa Nuestra Señora del Perpetuo Socorro, sede central.

- Municipio de Morelia: Institución Educativa Cervantes.

- Municipio de Belén de los Andaquíes: Institución Educativa Gabriela Mistral, sede Acevedo.

En dichas instituciones educativas se realizaron visitas de acompañamiento, inicialmente para aplicar el instrumento de investigación, así como también la revisión documental (PEI, planes de estudio grado Transición), y el acompañamiento para motivar la participación en la convocatoria de Ondas Colciencias, dependiente de la Vicerrectoría de Investigaciones de la Universidad de la Amazonia. 


\section{Metodología}

En primer momento, teniendo en cuenta los objetivos propuestos para la primera fase de la investigación, y en correspondencia con la investigación acción, se realizó el diagnóstico a través de la sistematización de los proyectos de investigación de III semestre, haciendo uso de la "rejilla síntesis de resultados pregunta de investigación" que alimenta la LINFA. En coherencia con el análisis realizado a esta información, se determinan dos criterios; tales como, contexto y egresado para focalizar las instituciones educativas que son objeto de investigación.

En un segundo momento, se procedió a la aplicación de la encuesta diseñada con el fin de identificar el conocimiento de los docentes acerca de las TIC y el uso que de ellas hacen como estrategia didáctica en los procesos de formación integral de la infancia. Para tal fin, se realizaron las respectivas visitas de las diferentes instituciones educativas focalizadas, en las cuales se logró recopilar información por medio de la observación, las conversaciones con docentes, directivos y obtener el registro fotográfico.

En tercera instancia, con el fin de identificar la manera en que las TIC están siendo incorporadas a los procesos de formación integral de la infancia, se hicieron las respectivas visitas a las diferentes instituciones focalizadas, para acceder a los Proyectos Educativos Institucionales (PEI) y a los planes de estudio del preescolar, grado Transición, y así poder realizar su respectivo análisis a partir del objetivo propuesto. Por consiguiente, en esta fase de la investigación se hizo trabajo de campo y revisión documental.

En un cuarto momento, para identificar la manera en que las TIC están siendo incorporadas a los procesos de formación integral de la infancia, se hizo el análisis de la información obtenida hasta el momento; esto permitió presentar una ponencia en un simposio a nivel internacional.

Finalmente, con el objetivo de formular la propuesta pedagógica de este proyecto de investigación para contribuir al mejoramiento de la inclusión de las TIC en los procesos escolares, se acompañó a las instituciones educativas focalizadas en jornadas pedagógicas para el conocimiento, diseño y aplicación del proyecto pedagógico de aula, apoyados en los estudiantes del VI y IX semestre de la Licenciatura en Pedagogía Infantil de la Universidad de la Amazonia. Asimismo, se continuó acompañando mediante jornadas pedagógicas para la evaluación de la propuesta. 


\section{Resultados}

Como resultado de las visitas, se logró, en primera instancia, hacer una caracterización de las instituciones educativas. En segundo lugar, se obtuvo a través de las encuestas diligenciadas y de las entrevistas informales con los docentes y directivos, además del registro fotográfico realizado, información relevante para cumplir con el objetivo propuesto en esta fase de la investigación, que consistió en identificar la relación existente entre el uso de las TIC y la intencionalidad pedagógica del desarrollo curricular. Por tanto, los hallazgos de investigación en esta primera fase son los siguientes:

Relacionado con el objetivo consistente en identificar el conocimiento que tienen los docentes y los padres de familia acerca de las TIC y el uso didáctico en los procesos de formación integral de la infancia, se diseñó y aplicó una encuesta que constó de trece preguntas estructuradas a partir de tres categorías de análisis: Concepciones, Estrategias y Entorno Familiar. En la primera categoría, se formularon cuatro preguntas con el fin de obtener información acerca de las concepciones de Ios docentes en torno a las TIC y su uso; en la segunda, otras cuatro preguntas para conocer las estrategias de incorporación de dichas tecnologías en el aula infantil con fines pedagógicos; bajo esta categoría se formuló una pregunta respecto al apoyo de los directivos para disponer de los medios tecnológicos de la institución; $y$, finalmente, se formularon otras cuatro preguntas para obtener información de parte de los docentes acerca de la percepción que tienen los padres sobre el uso de las tecnologías de la información y la comunicación en los procesos de formación de la infancia.

Por consiguiente, la fuente primaria de información fueron los docentes del grado Transición, incluso en el caso relacionado con el entorno familiar; dadas las dificultades temporales y espaciales para hacer una entrevista personalizada a los padres de familia, y considerando además que los docentes mantienen un contacto más directo con ellos, entonces se encuentran en la capacidad de proporcionar información pertinente. Sin embargo, hay que hacer la claridad que el objetivo principal de la encuesta estuvo dirigido al conocimiento y la manera en que los docentes del grado Transición hacen uso pedagógico de las TIC en el desarrollo curricular.

En este sentido, en las seis instituciones educativas visitadas, se diligenciaron en total diez encuestas, dando como resultado, en lo concerniente a la categoría sobre las concepciones de los docentes, una marcada diferencia entre aquellas que pertenecen al municipio de Florencia y las del departamento de Caquetá. Para 
el primer caso, se evidenció que los docentes tienen un concepto general sobre el significado de las TIC, puesto que claramente lo asocian con las tecnologías y sus mediaciones; en tal sentido, reconocen y hacen uso en el aula de algunas de ellas para desarrollar sus procesos de enseñanza y aprendizaje pese a que no tengan una intencionalidad pedagógica.

Mientras que en el segundo caso, solo lo relacionan de una manera muy general con las tecnologías, sin trascender por ende, en el uso pedagógico que implican estas en los procesos de enseñanza y aprendizaje en el aula escolar infantil, como lo afirma (Tripero, 2000).

Por otro lado, en cuanto a la aplicación de las estrategias para la incorporación de las TIC en el aula escolar, estas se determinaron a partir de la información obtenida, teniendo en cuenta los siguientes factores: perfil académico del agente educativo, modelos pedagógicos desarrollados en las instituciones educativas y la práctica docente en correspondencia a las orientaciones pedagógicas (Hernández, 2012).

Seguidamente, en lo relacionado con el entorno familiar, se puede ejemplificar la situación a partir de la respuesta dada por una docente del contexto rural, quien afirma que "Ios padres de familia no conocen el uso de las TIC, por lo tanto son indiferentes al tema; igualmente, en ocasiones se les ha invitado a que participen en cursos básicos de sistemas en la institución y su asistencia es escaza". Ello contrasta con la percepción en el contexto urbano del municipio de Florencia, donde "representa oportunidades de aprendizaje, actualización y diversión" (Rodríguez, 2011).

Mientras que en las instituciones educativas departamentales, se conoció que los padres de familia, aun cuando en términos generales poseen una percepción favorable acerca de las TIC, desconocen el uso pedagógico de estas, sobre todo lo relacionado con los medios de comunicación masiva, como la Internet y la televisión; como lo afirman los docentes. Es de aclarar que no fue esta la única estrategia para recoger información sobre el diagnóstico, puesto que en las visitas realizadas se hizo un registro personal de parte de cada investigador, con base en las conversaciones establecidas tanto con los directivos como los docentes y demás personas vinculadas a las instituciones. Como resultado, se identificaron otros aspectos de gran relevancia para este diagnóstico.

Es el caso por ejemplo de una situación en particular que al compartir entre el grupo de investigadores lo observado en las visitas a las diferentes instituciones, fue recurrente la negación del acceso a las salas de informática a los niños del nivel 
preescolar. Las razones de ello giran en torno al posible deterioro de los equipos de dichas salas por parte de los niños, la no adecuación de las salas para los niños de estas edades; situación que por supuesto obstaculiza, en cierto grado, la posibilidad para los docentes de preescolar de integrar en sus procesos de formación este tipo de tecnologías de comunicación (Papert, 1995).

Por consiguiente, se puede afirmar, en primer lugar, que en lo relacionado con el conocimiento de los docentes respecto a las TIC, depende de varios factores tales como: el perfil académico de los docentes, al igual que su trayectoria en el campo de la educación, la infraestructura tecnológica con que cuentan las diferentes instituciones, la disponibilidad de medios tecnológicos en el aula de preescolar y la modalidad o énfasis de las instituciones, entre otros. Entre los aspectos anteriormente mencionados, se evidenció una concepción generalizada de carácter preventivo, respecto al manejo que los niños del nivel preescolar le pueden dar a los equipos; principalmente de parte de los directivos, razón por la cual no se les permite el acceso. Esto genera, a su vez, que aquellos docentes interesados en integrar en sus procesos de formación este y otro tipo de tecnologías, tomen la iniciativa y lo hagan con sus propios recursos, como se evidenció en algunos casos particulares en instituciones ubicadas en el contexto rural.

En este sentido, un factor determinante para la no incorporación de las TIC a los procesos pedagógicos en la infancia, está relacionado con la designación de docentes de avanzada edad en el nivel preescolar. Esto puede llegar a ser un impedimento en determinados casos, mas no puede hacerse de ello una generalización. Igualmente, se identificó que en las instituciones que poseen la infraestructura y los recursos tecnológicos adecuados, no se desarrollan procesos de formación y actualización permanente del uso de las TIC; lo que conlleva a que los docentes desarrollen sus prácticas desde lo empírico y por iniciativa propia (Guerrero, 2011).

Retomando los hallazgos de la fase diagnóstica, también se identificó que en las prácticas de enseñanza desarrolladas tanto en los centros de atención a la infancia como en el aula escolar infantil, no se hace uso pedagógico de las TIC con el ánimo de crear nuevos modelos de educación que generen satisfacción e interés de aprendizaje en el niño; de igual forma, se encontró que las actividades que desarrollan con los niños tienen un propósito diferente a la formación o intencionalidad pedagógica, enfocándose a hacer uso de las TIC como una estrategia para recrear, entretener o distraer; aun cuando en la mayoría de las aulas y centros de atención a la infancia existen dispositivos tecnológicos de comunicación que pueden ser utilizados como herramientas de aprendizaje (televisor, computador, DVD, video 
beam, proyector de acetatos, grabadora, radio, prensa, emisora escolar, cámaras de video; e incluso, algunas instituciones tienen tablero digital, entre otros).

De manera que no se evidencia un plan de curso o proyecto pedagógico de aula encaminado a la utilización efectiva de estos medios para generar aprendizajes significativos en los estudiantes del grado Transición, por el contrario, se maneja una concepción equivocada relacionada con apreciaciones como la siguiente: "Ios niños, por ser pequeños dañan estos elementos"; en tal sentido, se desconoce los planteamientos de Dussel (2011) que afirma: "aprender y enseñar en la cultura digital es una necesidad actual".

En correspondencia con la fase de revisión documental para identificar la manera en que las TIC están siendo incorporadas a los procesos de formación integral de la infancia, se accedió a los Proyectos Educativos Institucionales (PEI) y a los Planes de Estudio del Preescolar, grado Transición, en los cuales se encontró un rasgo que comparten en común las diferentes instituciones educativas, en la mayoría definen en el componente teleológico del PEI, un modelo pedagógico de tipo constructivista.

El constructivismo ofrece un nuevo paradigma para esta nueva era de información y la comunicación; motivado por las nuevas tecnologías que han surgido en los últimos años. Con la llegada de estas tecnologías (wikis, redes sociales, blogs...), los estudiantes no solo tienen a su alcance el acceso a un mundo de información ilimitada de manera instantánea, sino que también se les ofrece la posibilidad de controlar ellos mismos la dirección de su propio aprendizaje (Vygotsky, 1978).

En este sentido, en el desarrollo del discurso argumentan identificarse como una comunidad educativa en la cual prima el trabajo en equipo, para generar en los estudiantes espacios propicios en la construcción del conocimiento, en los cuales el estudiante es sujeto activo. Sin embargo, haber encontrado este aspecto en común no representa en sí una novedad, puesto que este modelo constituye el paradigma predominante en la actualidad. Lo significativo del hallazgo reside en que este modelo y el humanista hacen parte de los pilares de la educación para los medios en la era de la información y la comunicación; es decir, los postulados de este modelo pedagógico, más allá de sus detractores o defensores, permiten la articulación de las TIC con los procesos de enseñanza-aprendizaje, siempre y cuando dicho modelo sea llevado a la práctica de manera consecuente; puesto que "desde la perspectiva vigotskiana, las tecnologías de la comunicación son los útiles con los 
que el hombre construye la representación externa que más tarde se incorporará o interiorizará mentalmente" (Vásquez, 2004).

Por consiguiente, los PEI de las diferentes instituciones educativas, de entrada, tendrían un pilar para la integración y articulación de las TIC a sus procesos de formación; no obstante, en el proceso de revisión y análisis no se encontró esta articulación, la cual debería verse reflejada, por ejemplo, en los proyectos transversales que, en correspondencia con la Ley General de Educación, en su artículo 14, se deberían desarrollar de acuerdo a las condiciones particulares de cada contexto educativo.

Esto puede ser quizás aún más llamativo en las instituciones educativas que han definido su modalidad en tecnología e informática o técnico en sistemas; aun cuando hay que reconocer las diferencias cualitativas entre adquirir una competencia en el manejo de las tecnologías y el uso de las TIC como estrategia pedagógica; la primera, está relacionada con las competencias que debe desarrollar el estudiante; mientras que la segunda, con la competencia del docente para hacer uso de las TIC con una intencionalidad pedagógica, la cual es el principal objeto de interés en esta investigación.

Desde esta perspectiva, hay que tener en cuenta además que el MEN y el Ministerio de las TIC propusieron desde el Plan Decenal de Educación, el objetivo fundamental a través del cual las instituciones educativas deben reorientar los procesos curriculares y dar respuesta a las necesidades de la era de la información y la comunicación, planteado de la siguiente manera: "Rediseñar proyectos educativos institucionales (PEI) y planes educativos municipales que incluyan el uso ético y pedagógico de las TIC, permitiendo mejorar los currículos orientados hacia los procesos investigativos, informativos y al desarrollo de las inteligencias cognitivas, sociales y prácticas" (MEN, 2006, p. 14).

Retomando lo que se puede llamar el eje articulador en el funcionamiento orgánico de los $\mathrm{PEI}$, correspondiente a los proyectos transversales, resalta el hecho de no haber encontrado ningún proyecto de este tipo que tuviera un énfasis 0 enfoque en las TIC. Por ejemplo, para el caso del Municipio de Florencia, en el año 2012 los docentes adscritos a la comunidad académica de preescolar, grado Transición, construyeron el "Plan de estudios de preescolar grado Transición" como documento de apoyo, estructurado en cuatro proyectos lúdico pedagógicos que pretenden integrar las dimensiones y competencias propias de este nivel, sustentado en los siguientes proyectos (docentes comunidad académica de preescolar, 2012): 
- Educando mi afectividad.

- Eco Ecología.

- Colombia el país en que vivo.

- Cuéntame un cuento (se menciona que "este proyecto va inmerso dentro de los otros proyectos de manera transversal").

Con ello, se pretende orientar la práctica docente fundamentada en proyectos de aula planteados desde tres ámbitos: ámbito de sí mismo, ámbito técnico científico y ámbito comunicativo en el mundo. Igualmente se menciona que el enfoque central de cada una de las actividades a desarrollar es el juego considerado como estrategia fundamental. En consecuencia, se resalta que los proyectos propuestos en el Plan de estudios de preescolar, grado Transición, pretenden fortalecer los proyectos pedagógicos obligatorios como son: educación para el ejercicio de los derechos humanos, educación sexual, medio ambiente, lúdicas, competencia ciudadana a través de la transversalidad pero el documento no es explícito con la incorporación de las TIC en el aula escolar infantil (Secretaría de Educación Municipal. Plan de estudios para preescolar en el grado Transición, 2011).

No obstante, se encontró que en la estructura propuesta para el desarrollo del proyecto de aula en lo que refiere a competencias, conocimiento esencial, desempeños y estrategias pedagógicas, no se incluyen las TIC para promover y acompañar el aprendizaje en el desarrollo de las competencias comunicativas, ciudadanas, matemáticas y científicas. De igual forma, se evidenció que en el contexto de la práctica docente no se aprovechan las potencialidades de las TIC en beneficio de los procesos educativos de infancia, como también, se desconoce el uso pedagógico de los medios tecnológicos de comunicación (Rodríguez, 2010).

Otro hallazgo significativo y que a su vez se encuentra en estrecha relación con los anteriores, es la desarticulación en los planes de estudio entre la propuesta curricular y el enfoque por competencias; dado que el común denominador hallado corresponde a que el diseño curricular del plan de estudios presenta una estructura lineal, dividido en áreas y asignaturas; fundamentado especialmente en el desarrollo por dimensiones; todas estas características contrastan con la intencionalidad discursiva de aplicar tendencias modernas de enseñanza y aprendizaje en la infancia (Blatchford, 2004). Este hallazgo se puede considerar una consecuencia 
directa de la desactualización de los PEI, lo cual repercute, de una u otra forma, en la práctica docente; dado que en algunos casos se pudo evidenciar la inexistencia de coherencia entre el discurso y la práctica pedagógica, salvo algunas excepciones, en el sentido que esta corresponde a la aplicación de modelos normativos e instructivos; cuando ella debería estar inserta en los fundamentos de la escuela activa y el constructivismo pedagógico.

El planteamiento teórico que dio lugar a la propuesta constructivista que se implementa hoy fue diseñado por César Coll, esta teoría es atribuida a la idea de Piaget y Vygotsky. Según la teoría psicológica cognitiva, la interrelación profesor/alumno es una estructura comunicativa o proceso de interacción en el que interviene: la actividad constructiva de los alumnos y el papel del mediador del profesor. Según esta interpretación, aunque es el alumno el que construye su aprendizaje, no lo efectúa solo, en este sentido el verdadero papel del profesor consiste en actuar de intermediario entre los contenidos del aprendizaje y la actividad constructiva que despliegan los alumnos para asimilarlos. El profesor, en la teoría constructivista, es un intermediario. Pero, ¿cómo se ejerce la influencia?, ¿cómo consigue el profesor incidir en la actividad constructiva del alumno con el fin de ayudarle a asimilar los contenidos escolares? Aquí entran en juego los planteamientos de Vygotsky. Se intenta con ello situar la actividad constructiva psíquica del alumno en el entramado de las relaciones sociales (Vygotsky, 1988).

En correspondencia con lo anterior, los hallazgos confirman algunas de las expectativas e hipótesis de las cuales surgió esta investigación.

Que los docentes de infancia tienen un concepto general sobre el significado de las TIC, lo asocian con las tecnologías y sus mediaciones, sin transcender en el uso pedagógico que implican estas en el proceso de enseñanza y aprendizaje en el nivel preescolar, además, las entidades territoriales y las instituciones educativas no realizan procesos de formación docente y actualización permanente en el uso pedagógico de las TIC, lo que conlleva al desarrollo de las prácticas educativas desde lo empírico sin intencionalidad pedagógica y ausencia de los procesos de articulación a los proyectos pedagógico institucionales (Agudelo, 2014, p. 1).

Con respecto a la cuarta fase de esta investigación, y como resultado de las visitas a las instituciones educativas focalizadas, se acordó trabajar conjuntamente 
para participar en la convocatoria 2013 del proyecto "Fortalecimiento del Programa Ondas Colciencias en el Caquetá" Fondo de Ciencia, Tecnología e Innovación del Sistema General de Regalías, en la categoría de proyectos abiertos, la cual se constituye en estos momentos en la mejor opción para realizar un trabajo colaborativo con las instituciones educativas focalizadas, con miras a generar procesos de formación integral a la infancia, por medio de una articulación adecuada y pertinente de las TIC a estos procesos. Sin embargo, para poder dar cumplimiento a ello, se convierte en una condición indispensable la participación activa de los docentes de preescolar de las diferentes instituciones. Es de aclarar que con esta propuesta se proyecta la estrategia de acción del presente proyecto de investigación.

Para concluir el desarrollo de las respectivas fases del proyecto de investigación y contribuir al mejoramiento de los procesos de enseñanza y aprendizaje respecto a la incorporación de las TIC en la infancia, se acompañó a las instituciones educativas focalizadas para formular propuestas pedagógicas que aporten al mejoramiento de la inclusión de las TIC en los procesos escolares a través de jornadas pedagógicas, donde la metodología empleada en el desarrollo de esta actividad particularmente se enfatizó hacia el conocimiento, conceptualización, caracterización y fases de un proyecto pedagógico de aula; estructurado a partir de una serie de secuencias didácticas que retoman el uso pedagógico de las TIC como una estrategia transversal en la formación integral de la infancia. Es de aclarar que en este proceso de aplicación de la estrategia se contó con el apoyo de estudiantes de VI y IX semestre de la Licenciatura en Pedagogía Infantil, quienes en su momento se encontraban realizando la práctica pedagógica de intervención.

A modo de reflexión con respecto a este proyecto de investigación se concluye que:

Hoy se vive en un constante cambio, un reto permanente a comprender que socialmente la formación integral del ser humano está directamente relacionada con la articulación que se genere entre tres aspectos fundamentales: Información, Conocimiento e Inteligencia, que implican un nuevo orden conceptual y pragmático de la relación educación y TIC (Tedesco, 2000).

En particular el planteamiento sociocultural vigotskiano hoy recobra significación, en tanto que aporta una base epistemológica que permite replantear y dar sentido a muchas prácticas, enfoques educativos y teorías de aprendizaje tradicionales a partir de la promoción del pensamiento crítico, cuestionador y reflexivo. 
Dicho pensamiento convoca a los docentes a considerar las nuevas tecnologías de la información como instrumentos de mediación pedagógica que permiten acceder a nuevos conceptos, conocimientos, una nueva comprensión de hechos, fenómenos actuales para dar respuesta a los intereses y necesidades de formación del contexto (Rodríguez, 2011), dejar de lado el uso de las TIC es privar a la educación de posibilidades insospechadas y dejar de lado una excelente oportunidad para que los maestros innoven. Al respecto esta investigación identificó que aún en los docentes falta mayor compromiso para incorporar el uso pedagógico de las TIC en el proceso de formación integral de la infancia.

Asimismo esta investigación logró vincular a padres de familia en la implementación del proyecto pedagógico de aula, mediante acciones relacionadas con el uso pedagógico de herramientas virtuales de aprendizaje, lo que permitió de alguna manera generar conciencia en los padres de familia sobre la importancia de acompañar los procesos de aprendizaje de los niños en relación a la necesidad de regular el uso de los medios de comunicación y la información que reciben los niños a través de medios como la televisión y la Internet; facilitando de esta manera la intencionalidad pedagógica de las TIC en la formación integral del niño (Carlos, 2000).

Se identifica importante considerar en los procesos de enseñanza la realidad indiscutible que avoca al docente en la actualidad a incorporar en su práctica pedagógica nuevas estrategias acordes a la circunstancia; implica reconocer que los niños nacen en la Era de las Tecnologías o Era Digital (Tripero, 2000), y con ellas conviven de forma espontánea, con el interés constante de explorar, comprender y dominar la tecnología que tienen a su alcance y ponerla al servicio de sus necesidades; por lo tanto, la escuela no debe estar aislada o desintegrada de ellas.

En algunas instituciones educativas, tras la implementación de la propuesta pedagógica, se logró sensibilizar a directivos institucionales respecto a la importancia de permitir el ingreso y uso de las salas de informática al nivel preescolar; para el caso de las instituciones que poseen esta infraestructura, para las otras instituciones, se logró la organización del rincón tecnológico para implementar de esta manera las herramientas virtuales de aprendizaje en la formación de los niños.

Se evidenció que los hallazgos de esta investigación se constituyen en un factor común en otras regiones de Colombia, como se constató en las interacciones con otras comunidades educativas en el marco del Simposio Internacional de Educación y Pedagogía; escenario propicio para la divulgación de hallazgos de investigación en el cual se participó. 
Teniendo en cuenta la necesidad de acompañamiento pedagógico que requieren las instituciones educativas en el nivel preescolar, el Comité de Investigaciones de la Universidad de la Amazonia, emite como concepto final en el segundo periodo académico de 2014 lo siguiente: es necesario y pertinente que esta investigación sea continuada desde la Universidad de la Amazonia para avanzar en nuevas fases que permitan la generación de alternativas pedagógicas en el trabajo articulado con los docentes, a través de metodologías de acompañamiento en el aula, posiblemente con estudiantes de la Licenciatura en Pedagogía Infantil. Por esta razón el presente proceso de investigación iniciará la fase dos en el segundo periodo académico del 2015.

\section{Referencias Bibliográficas}

Agudelo, E. R. (2014). Informe final El uso de las TICs en los procesos de formación integral de la infancia. Florencia: Universidad de la Amazonia.

Barbero, J. M. (2005). Restos culturales de la comunicación a la educación. Nueva sociedad, 169. Cali, Colombia.

Blatchford, J. S. (2004). Nuevas tecnologías para la educación infantil y primaria. Madrid: Ediciones Morata.

Buckingham, D. (2002). Crecer en la era de los medios electrónicos. Madrid: Ediciones Morata.

Carneiro, R. L. (2010). El uso generalizado de las TIC impacta a la sociedad, la economía y la cultura. Bogotá: Elsevier.

Celi, E. H., et al. (2012). Documento maestro. Florencia: Uniamazonia.

Crook, C. (1998). Una introducción al aprendizaje colaborativo. Conexión Universidad EAFIT, 9-10.

Docentes comunidad académica de preescolar. (2012). Plan de estudios de preescolar, grado Transición. Florencia, Caquetá: Secretaría de educación municipal.

Dussel, I. (2011). Aprender y enseñar en la cultura digital. En Ruta Maestra. Buenos Aires: Fundación Santillana.

Escudero, J. M. (2000). La educación y la sociedad de la información: cuestiones de contexto y bases para un dialogo necesario. Mérida: Universidad de Murcia Junta de Extremadura.

Guerrero, A. (2011). La integración de la tecnología en el currículo. En A. p. clases, Ruta Maestra. Santillana. 
Hernández, I. (2012). Ruta Maestra: La necesidad de orientar el uso didáctico de las nuevas tecnologías. Bogotá: Santillana S.A.

Hernández, I. (2012). Ruta Maestra. Bogotá: Santillana.

Miranda, J. V. (2004). La comunicación en la escuela infantil, algunas consideraciones teóricas. Madrid: Universidad Complutense.

Papert, S. (1995). La máquina de los niños: replantearse la educación en la era de los ordenadores. Paidós Ibérica S.A.

Prensky, M. (2001). Nativos e inmigrantes digitales - cuadernos SEK 2.0 - Aprender a pensar. Albatros.

Rodríguez, F. V. (2011). La necesidad de orientar el uso didáctico de las nuevas tecnologías. En Ruta Maestra. Bogotá: Santillana.

Rodríguez, J. R. (2010). Investigaciones sobre buenas prácticas con tecnologías de la información y la comunicación. En Ruta Maestra. Málaga: Ediciones Aljibe.

Tapscott, D. (1998). Creciendo en un entorno digital: la generación Net. McGraw-Hill.

Tedesco, J. C. (2000). Escuela y Cultura: una relación conflictiva. Buenos Aires: IIPE.

Tripero, T. d. (2000). Tecnologías de la Comunicación y del Aprendizaje en la Era Digital: de la sacralización y la veneración a las dificultades para su implantación. Huerga \& Fierro - Programa de tercer ciclo del Dpt Universidad Complutense. Programa de Tercer Ciclo del Dpt, p. 1-7.

Vygotsky, L. (1988). El desarrollo de los procesos psicológicos superiores. Interacción entre aprendizaje y desarrollo. México: Grijalbo. 